\title{
Etude du cycle biologique de deux Limnephilidae : Limnephi- lus minos et Limnephilus germanus (Trichoptera)
}

\author{
C. Denis 1 \\ H. Malicky²
}

Mots clés: Trichoptera, Limnephilidae, cycle biologique.

Le cycle biologique des deux espèces a été étudié dans la nature et au laboratoire.

L. minos vit en Crète, dans des lagunes saurnâtres et à une température de $15^{\circ} \mathrm{C}$. Les différents stades du développement sont présents pendant toute l'année. Des élevages ont montré que l'espèce était bivoltine et pouvait se développer en eau douce et à différentes températures.

L. germanus vit en Europe Centrale. En Autriche, les larves habitent dans des étangs gelés en hiver. Les adultes sont essentiellement automnaux. L'espèce, normalement univoltine, est bivoltine en laboratoire à $10^{\circ}$ ou à $15^{\circ} \mathrm{C}$. Dans la nature elle doit subir une longue quiescence hivernale à l'état d'embryon ou de jeune larve.

A l'émergence, les femelles des deux espèces sont immatures, mais ensuite leur développement génital est direct. Par contre, les femelles des autres espèces de Trichoptères, immatures à l'émergence, subissent une diapause estivale.

A study of the biological cycle of two species of Limnephilidae : Limnephilus minos and L. germanus Trichoptera.

Keywords : Trichoptera, Limnephilidae, biological cycle

The biological cycle of the two species was studied in the field and in the laboratory.

L. minos lives in Crete, in brackish lagoons at a constant temperature of about $15^{\circ} \mathrm{C}$. All stages are present throughout the year. Rearing experiments have shown that it is bivoltine and will develop also in fresh water and at different temperatures.

L. germanus lives in Central Europe. In Austria the larvae live in ponds which freeze in winter. The adults are essentially autumnal. This species, which is normally univoltine, is bivoltine in the laboratory at $10^{\circ}$ or $15^{\circ} \mathrm{C}$. In the field it undergoes a long winter quiescence as an embryo or young larva.

At emergence the females of both species are immature, but then their reproductive systems develop directly. In contrast, the females of other species of Trichoptera which are immature at emergence, undergo a summer diapause.

\section{1. - Introduction}

Sous les latitudes moyennes de l'Europe, les Limnephilidae dont le cycle biologique est connu sont en général univoltins et beaucoup subissent une diapause en été. Dans un premier cas, plusieurs Limnephilus, Grammotaulius nigropunctatus ( = atomarius), Glyphotaelius pellucidus (Novak \& Sehnal 1963 1965), les adultes émergent dès le printemps, les

\footnotetext{
1. Laboratuire de Biologie animate Ier Cycle. Université de Rennes, B.P. 25 A, F. 35042 Rennes, France.

2. Biologische Station Lunz, A-3293 Lunz am See, Autriche.
}

femelles sont alors immatures et elles ne pondent qu'en automne. Dans un second cas, Anabolia furcata (Novak 1960), A. nervosa (Denis 1979 et 1980) Halesus radiatus (Denis 1979), les adultes émergent en automne, les femelles sont pratiquement prêtes a pondre et ce sont les larves, en fin de croissance, qui subissent un repos estival. Toutefois, dans un troisième cas, Drusus annulatus (Gower 1965 et 1973), l'ensemble du développement est direct et les femelles, à l'émergence, sont en fin de maturité génitale. Selon la température de l'eau où vivent les larves, l'espèce est bivoltine et les adultes présents pendant toute l'année, ou elle est univolt ine et les adultes ne sont visibles qu'en été. 
Limnephilus minos et L. gemanus sont deux espèces très différentes par la phénologie. A l'aide de données recueillies dans la nature et d'élevages en laboratoire, nous avons étudié le cycle biologique de chaque espèce afin de savoir s'il se déroulait conformément à l'un des cas précédemment cités.

\section{2. - Données recueillies dans la nature}

Limnephilus minos Malicky n'est connu que dans l'île de Crète où l'espèce fut découverte en 1969. Et c'est en 1972 que les premières larves furent trouvées, en grand nombre, dans la lagune saumâtre de Georgioupolis (fig. 1). Dans les sources de Georgioupolis la conductivité de l'eau varie entre 2900 et $4500 \mu \mathrm{S} 20$, ce qui correspond à des concentrations de 2 à $3 \%$ en matières minérales. Nous avons alors pensé que les larves se confinaient dans les sources saumâtres (Malicky 1974). Cette opinion fut apparemment confirmée par la découverte des larves dans la rivière Kiliaris, près de Kalyves, un ruisseau large où la concentration en matières minérales est également d'environ $3 \%$, excepté dans les périodes d'inondation où elle diminue jusqu'à $0,3 \%$ en raison de la dilution. Toutefois, l'espèce n'habite pas dans les eaux à plus forte salinité. Cela a été vérifié dans une source saumâtre située au sud de la ville d'Agios Nikolaos (salinité de 5 à $6 \%$ ) et dans la source Almiros, à l'ouest de la ville d'Iraklion (salinité de $10 \%$ ). Une salinité de 2 à $3 \%$ - qui est inférieure à la limite de tolérance pour les animaux dulçaquicoles (Malicky 1974) - permet donc aux larves

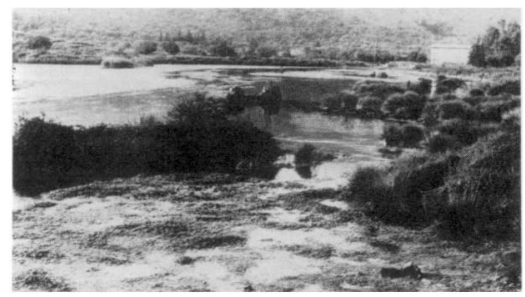

Fig. 1. La lagune de Georgioupolis en Crete. Devant on voit la végétation dense, submergèe d'Apium nodiflorum, plante dont les larves de Limnephilus minos se nourrissent. Les sources saumâtres se trouvent hors de l'image, aux pieds du photographe. de $L$. minos de vivre normalement, tandis qu'un taux de salinité supérieur doit être défavorable. Par contre la présence d'une certaine salinité n'est pas nécessaire. En effet, l'espèce fut retrouvée plus tard en Crète orientale entre les villages d'Agios loannis et de Schinokapsala, dans un petit ruisseau dont la salinité n'est que de $0,1 \%$. Enfin nous avons constaté, en laboratoire, que les larves se développaient parfaitement en eau douce.

Dans la lagune de Georgioupolis les larves vivent dans la partie basse de l'embouchure des sources saumâtres qui sortent des roches karstiques; là elles subissent le flux et le reflux (variations d'un quart de mètre environ). Les fourreaux des larves sont couverts de graviers, de particules végétales et de coquilles de Mollusques souvent vivants. Les pontes gélatineuses sont fixées sur des feuilles d'Apium nodiflorum ainsi que sur des feuilles et des racines surplombantes de Juncus sp (Cf. heldreichianus) régulièrement mouillées par le flux. Les larves se nourrissent de feuilles vertes immergées d'Apium nodiflorum. Mais là où cette plante est absente la nourriture des larves est inconnue. Dans le Kiliaris, elles vivent sur des Potamogeton sp. et s'en nourrissent probablement.

Tous les stades du développement sont présents pendant toute l'année. La durée des différents stades n'a pas pu être déterminée dans la nature.

Les sources saumâtres de Gieorgioupolis ont une température constante de $15^{\circ} \mathrm{C}$ pendant toute l'année ; les variations se situent entre $14^{\circ}, 9$ et $15^{\circ}, 2$, et il n'a été enregistré qu'une seule fois une température de $15^{\circ}, 6$. Dans le ruisseau près d'Agios Ioannis la température ne varie qu'entre $13^{\circ}, 2$ et $14^{\circ}, 8$. Dans la rivière Kiliaris la température varie entre $12^{\circ}$ et $18^{\circ}, 1$ mais elle est influencée par les fluctuations du débit d'eau dues aux inondations; en fait pendant la majeure partie de l'année la température de l'eau se situe entre $14^{\circ}$ et $16^{\circ}$. Les autres sources saumâtres mentionnées ont également des températures constantes (Almiros, $16^{\circ}$ à $16^{\circ}, 2$ et Agios Nikolaos, $14^{\circ}, 4$ à $14^{\circ}, 8$ ) mais leur salinité est trop élevée pour $L$. minos. Par contre, la plupart des ruisseaux de Crete subissent des variations thermiques assez fortes au cours de l'année et l'espèce ne les fréquente pas. Les minima se situent aux environs de $10^{\circ}$ en hiver et les maxima autour de $22^{\circ}$ en été. Le facteur clé pour la présence de l'espèce serait 
donc une température à peu près constante et voisine de $15^{\circ} \mathrm{C}$. Les localités où $L$. minos a été trouvé sont indiquées sur la figure 2.

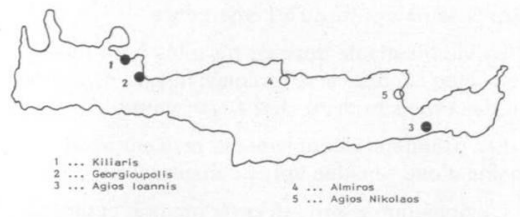

Fig. 2. Emplacement des localités où $L$ minos vit en Crète (points noirs).

Limnephilus germanus Mc Lachlan a une vaste aire de répartition dans les Alpes septentrionales et au nord de l'Europe. Il existe des données pour la Haute et la Basse Autriche, la Styrie, le Tyrol, et le Vorarlberg et pour le Sud de la Bavière. Dans les environs de Lunz (sud-ouest de la Basse Autriche) les larves vivent dans des étangs à truites traversés par un ruisseau. Le biotope caractéristique est constitué par la partie du ruisseau qui traverse l'étang. là où la vitesse du courant est réduite mais où l'oxygénation est bonne. Ce type de biotope est souvent couvert de macrophytes tels que Carex, Phragmites, Scirpus etc... Les larves vivent dans des endroits bien oxygénés mais semblent éviter le courant. Nous n'avons jamais trouvé de larves dans les ruisseaux eux-mêmes, ni aucun adulte sur la végétation qui les borde. L'étang de Rohrwiesteich, situé à $18 \mathrm{~km}$ au sud-est de Lunz et à une altitude de 900 mètres, héberge une abondante population de $L$. germanus. Sur la photo (fig. 3) on voit l'étang, lui-même et, bien distinct de lui, le ruisseau qui serpente parmi les macrophytes.

La nourriture des larves est inconnue dans la nature.

On sait depuis longtemps que $L$. germanus est une espèce automnale. Les adultes ont été capturés principalement entre début Septembre et mi-Novembre avec un maximum en fin Octobre. On connaît toutefois quelques exceptions. Ainsi sur 290 exemplaires récoltés dans les Alpes du Nord, 6 ont été capturés bien avant l'automne : un le 9 Août, 3 pendant la troisième décade de Juillet, un le 6 Juin et même un le 22 Mars.

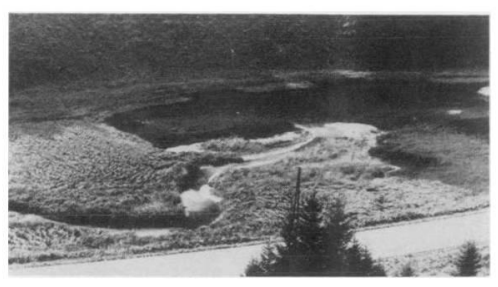

Fig. 3. L'étang à truites a Rohrwiesteich en Basse Autriche, biotope typique de Limnephilus germanus. Les flèches indiquent la zone où des larves ont été trouvées en Juin 1982.

Il faut noter, par ailleurs, qu'à la chasse au filet, dans la végétation qui borde les biotopes, on trouve beaucoup plus de femelles que de máles. Ceci tient peut-être au fait que ces derniers se dissimulent mieux à la base des végétaux. En effet, dans les pièges lumineux les nombres $d$ 'individus capturés sont identiques pour les deux sexes, et on note une légère protandrie.

Les œufs sont pondus en Octobre et Novembre. Du fait que les adultes n'apparaissent en général qu'en automne, la vie aquatique de l'espèce se déroule durant la majeure partie de l'année. Mais nous avons peu de données sur cette partie du cycle. En effet, il a été difficile de trouver des larves. En 1982 nous les avons vainement cherchées en Février, en Mars et en Mai. Par contre vers la fin Juin nous avons découvert quelques dizaines de larves dont la plupart étaient à l'avant dernier stade. Elles se trouvaient à l'endroit indiqué par deux flèches sur la figure 3. De nouvelles recherches en Juillet et en Août, au même endroit, furent vaines. Mais ceci peut s'expliquer par la longue sécheresse de 1982 au cours de laquelle l'eau baissa et ne fut plus agitée dans la zone précédemment indiquée provoquant, en cet endroit, la précipitation de la vase et la prolifération des algues filamenteuses. Dans ces conditions de nombreuses larves ont dû périr. En effet, durant l'automne 1982 les adultes ont été beaucoup moins nombreux que les années précédentes.

N'ayant trouvé les larves qu'à une seule époque de l'année, nous ignorons comment se déroule la totalité de la phase aquatique et si les animaux sont sédentaires ou effectuent des migrations, notamment en fonction des variations du niveau de l'eau 
liées au régime irrégulier des pluies. Il était donc impossible de determiner exactement les conditions de température auxquelles les embryons et les larves sont soumis. Toutefois des enregistrements effectués pendant toute l'année nous renseignent sur l'amplitude des variations thermiques dans des eaux similaires de la région (Malicky 1978). Au cours de la saison froide (Novembre à Mai) ces étangs sont recouverts de glace pendant longtemps et, même sans cette couverture, la température et le niveau de l'eau varient irrégulièrement en fonction des conditions météorologiques. La température se situe alors entre 5 et $20^{\circ} \mathrm{C}$ et peut passer d'une extrême à l'autre en quelques heures.

\section{3. - Etudes en laboratoire}

Les premiers élevages ont été entrepris à partir de masses ovigères obtenues en capturant des femelles prêtes à pondre.

Durant tout leur cycle biologique les deux espèces ont été maintenues à une température de $15^{\circ} \mathrm{C}$ et à la photopériode naturelle. Les dispositifs d'élevage des larves et des adultes ont déjà été décrits (Allegret \& Denis 1972, Denis 1972). Les larves de $L$. minos ont été alimentées aussi bien avec de l'herbe verte (ray-gras) qu'avec des feuilles mortes tandis que les larves de $L$. germanus se sont nourries uniquement de feuilles mortes.

Les premiers élevages de $L$. minos ont commencé en Avril 1978, ceux de L. germanus en Octobre 1977.

\section{Résultats}

Dans les conditions du laboratoire, les cycles biologiques des deux espèces sont à peu près identiques. Ils durent de cinq à six mois.

Le développement embryonnaire commence aussitôt et dure environ 15 jours.

Les deux espèces passent par 5 stades larvaires. La durée globale du développement larvaire est de trois à quatre mois, mais peut se prolonger au-delà pour certaines larves de L. germanus. Chacun des quatre premiers stades se déroule en une dizaine de jours au minimum, mais les variations individuelles sont importantes à partir du troisième stade, surtout dans le cas de $L$. germanus.
La métamorphose se produit en trois semaines environ. Il s'écoule une semaine entre la confection de la logette nymphale (obturation des deux extrémités du fourreau) et la mue nymphale, puis deux autres semaines jusqu'à l'émergence.

La vie imaginale dure de un mois à un mois et demi chez L. minos et se prolonge jusqu'à deux mois ou deux mois et demi chez L. germanus.

Les premiers accouplements peuvent avoir lieu moins d'une semaine après l'émergence.

L'oviposition se produit entre un mois et un mois et demi après l'émergence chez $L$. minos et les femelles meurent quelques jours plus tard. Chez $L$. germanus l'oviposition peut avoir lieu entre 10 jours et plus d'un mois après l'émergence. En outre, les femelles demeurent vivantes pendant un mois à un mois et demi après avoir pondu. Deux femelles ont déposé chacune, à un mois d'intervalle, deux masses ovigères.

Lorsqu'ils émergent les mâles des deux espèces ont achevé leur maturation génitale. Toutefois, les glandes accessoires de leur appareil génital ne sont qu'à demi ou aux deux tiers remplies de sécrétion. Les femelles, quant à elles, sont immatures. La zone basale des ovarioles - le vitellarium - contient des ovocytes en prévitellogénèse avancée. Les glandes collétériques sont petites et commencent à peine à élaborer leur sécrétion. La vitellogénèse débute quelques jours après l'émergence, ce qui coïncide à peu près avec l'apparition des premiers accouplements.

\section{4. - Discussion}

\section{1. - Cas de Limnephilus minos}

L'élevage en laboratoire dans l'eau de la ville montre que les larves vivent aussi bien en eau douce qu'en eau saumâtre.

La température de $15^{\circ} \mathrm{C}$ utilisée dans les élevages correspond sensiblement à celle de l'eau où vivent habituellement les larves. Par ailleurs, nous avons vérifié, par des expériences complémentaires, que la durée de la photopériode était sans effet sur les différents stades du développement. Des résultats obtenus en laboratoire nous pouvons donc deduire que $L$. minos présente, dans la nature, deux générations par an. Mais elles ne sont pas synchronisées. 
Nous avons également cherché à déterminer une action éventuelle de la température sur les différentes phases du cycle biologique. A une température voisine de $20^{\circ} \mathrm{C}$ le cycle se déroule normalement, sensiblement à la même vitesse qu'à $15^{\circ} \mathrm{C}$; le développement larvaire tend toutefois à s'accélérer. A une température de $10^{\circ} \mathrm{C}$, le cycle se déroule encore normalement. La chronologie du développement des femelles adultes est à peine modifiée, seule la vie aquatique es t plus longue, elle dure alors entre six et sept mois. Les élevages en laboratoire ne semblent donc pas confirmer le rôle de la température en tant que facteur limitant pour l'espèce. Si dans la nature des larves vivent constamment dans une eau à $15^{\circ} \mathrm{C}$, ce n'est donc pas en raison d'une nécessité physiologique ; mais cette température favorise probablement le maintien des autres conditions nécessaires aux animaux.

\section{2. - Cas de Limnephilus germanus}

Les conditions de température utilisées en laboratoire $\left(15^{\circ} \mathrm{C}\right)$ diffèrent beaucoup de celles que l'espèce subit dans la nature, et le cycle obtenu expérimentalement est donc très différent du cycle normal.

Les problèmes posés par le cycle biologique de $L$. germanus sont les suivants :

- Comment l'espèce passe-t-elle l'hiver, lorsque l'eau est à une température voisine de $0^{\circ} \mathrm{C}$ ?

- Les adultes émergeant en automne, l'espèce subit-elle une diapause estivale à la fin du développement larvaire ou pendant la nymphose?

a) Question de la période hivernale du cycle

Nous n'avons pu réaliser des expériences à une température voisine de $0^{\circ} \mathrm{C}$, mais à $5^{\circ} \mathrm{C}$ les embryons et les jeunes larves se développent déjà très lentement. Nous pensons donc que dans les conditions hivernales de la Basse Autriche (température de 0 à $2^{\circ} \mathrm{C}$ ), le développement de l'espèce est pratiquement stoppé.

Lorsque les pontes sont déposées en Octobre, les œufs peuvent éclore et les larves commencer à se développer avant le refroidissement important de l'eau. Au cont raire, lorsque les pontes sont déposées en Novembre, la température de l'eau atteint sûrement son minimum avant la fin de l'embryogénèse et les ceufs ne doivent éclore qu'au printemps. D'une manière générale, le cycle doit ètre à peu près stoppé entre Novembre et Mai, soit durant la vie embryonnaire, soit au début de la vie larvaire.

b) Question d'une éventuelle diapause estivale à la fin de la croissance larvaire ou pendant la nymphose.

Une telle diapause est induite lorsque les jeunes stades larvaires ont été soumis à l'action d'une photoperiode de type hivernal (Denis 1980). Nous avons donc élevé des larves à une photopériode de 12 heures et nous les avons exposées à une photopériode de 16 heures vers la fin de leur croissance. Nous n'avons constaté aucun arrêt du développement ultérieur. Nous avons également vérifié que les femelles adultes n'étaient pas sensibles à l'action de la photopériode.

Nous pouvons déduire de cette étude que $L$. germanus a un cycle biologique annuel sans diapause estivale. Les mois d'été pendant lesquels la température de l'eau est la plus élevée sont ceux où la majorité de la croissance larvaire et la métamorphose ont lieu, et rapidement.

\section{3. - Comparaison entre les cycles des deux espè- ces et ceux des autres Limnephilides}

Les élevages en laboratoire ont montré que les cycles de $L$. minos et de $L$. germanus peuvent se dérouler en moins de six mois, ce qui permet d'obtenir expérimentalement deux générations annuelles, comme cela a été le cas pour d'autres Limnephilides (Denis 1972 a et b).

Le cycle de $L$. minos se déroule sensiblement comme celui de Drusus annulatus, en eau tempérée (Gower 1965), avec deux générations par an. Toutefois, si les femelles de $D$. annulatus ont pratiquement achevé leur maturation génitale, quand elles émergent, celles de $L$. minos sont encore immatures et en prévitellogenèse.

Le cycle de $L$. germanus diffère de celui des autres Limnéphilides à adultes automnaux sur plusieurs points :

- En raison du long ralentissement hivernal, la croissance larvaire est essentiellement estivale, à l'époque où les autres espèces automnales sont en diapause larvaire.

- Lorsqu'elles émergent les femelles des espèces automnales sont en général pratiquement prêtes à pondre, tandis que celles de L. germanus sont immatures et en prévitellogenèse. 
A l'émergence les femelles de $L$. minos et de $L$. germantus sont dans le même ćtat d'immaturité génitale que celles des autres Limnephilus, mais qui, elles, subissent une diapause estivale.

Enfin il faut souligner la possibilité que les femelles de $L$ germanus ont de pouvoir pondre deux fois à un mois d'intervalle. Ceci est très différent du cas des femelles qui déposent leurs oufs en deux ou trois masses ovigères pondues à quelques heures d'intervalle. Le cas de $L$. germanus implique le déroulement de deux cycles successifs complets de vitellogenèse. Ce fait est très rare chez les Trichoptères. Habituellement lorsque les femelles ont achevé leur premier cycle de vitellogenèse, un second cycle commence, mais en général il ne va jamais jusqu'à son terme (Denis \& Le Lannic 1977).

\section{Remerciements}

Nous remercions Madame Rault pour son aide technique concernant l'entretien des élevages.

\section{Travaux cités}

Allegret (P.) \& Denis (C.). 1972. - Dispositif pour l'élevage d'insectes aquatiques à température constante. Ann. Hydrobiol, 3 (I) : 65-67.
Denis (C.). 1972 a. - Etude du cycle biologique de Limnephilus lumatus (Trichoptera, Limnephilidae). Obtention de deux géné rations annuelles. Bull. Soc. Sci. Bretagne, 47 : 33-38.

Denis (C.). 1972 b. - Etude au laboratoire du cycle biologique de Arabolia nervosa (Trichoptera, Limnephilidae). Bull. Soc. Sci. Bretagne, 47 : 43-48.

Denis (C.) \& Le Lannic (J.). 1977. - Les modalités de la vitellogenèse chez les Trichoptères. Ann. Zool. Ecol. anim., 9 (4) : 627-635.

Denis (C.). 1979. - Comparaison entre la diapause larvaire che? Anabolia nervosa Curtis et Halesus radialus Curtis (Trichop. tera, Limnephilidae). Annls Limnol., 14 (3) : 215-224.

Denis (C.). 1980. - Action de la photopériode sur le cycle biologique et la diapause d'Anabolia mervosa Curtis (Trichoptera, Limrephilidae). Annis Limnol., 16 (3) : 191-202.

Gower (A.M.). 1965. - The life cycle of Drusus annulatus Steph. (Trich. Limnephilidae) in watercress beds. Entomologist's mon. Mag., $101: 133-141$.

Gower (A.M.). 1973, - The life cycle and larval growth of Drusus annularus Stephens (Trichoptera, Limnephilidae) in a mountain stream. J. Enzt. (A), 47 (2) : 191-199.

Malicky (H.). 1974. - Eine im marinen Gezeitenbereich lebende europaïsche Trichopterenlarve. Arch. Hydrobiol. 73 ; $266-269$.

Malicky (H.). 1978. - - Der Temperaturgang einiger niederösterreichischer Gebirgsbäche. Wetter und Leben (Wien) $30: 170-183$.

Novak (K.). 1960. - Entwicklung und Diapause des Köcherfliegenlarven Anabolia furcaza Br. (Trichoptera). Acta Soc. ent. Cechoslov., $57: 207-212$.

Novak (K.) \& Sehnal (F.). 1963. - The development cycle of some species of the genus Limnophilus (Trichoptera). Cas. csl. Spol. Ent. $60: 68.80$.

Novak (K.) \& Sehnal (F.). 1965. - Imaginaldiapause bei den іл periodischen gewässern lebenden Trichopteren, XII th. Intern. Congress of Entomology, London. 